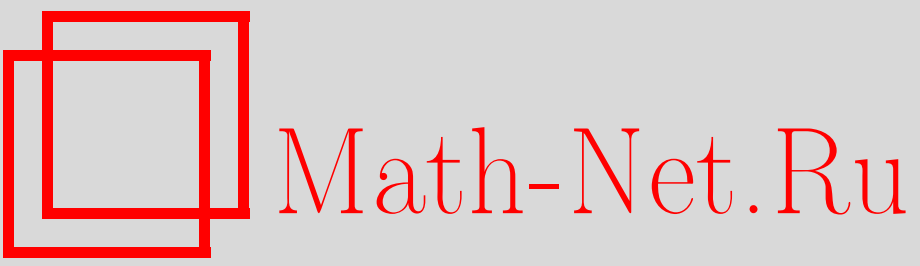

Ю. И. Скалько, С. Ю. Гриднев, Обобщенная задача Римана о распаде разрыва с дополнительными условиями на границе и ее применение для построения вычислительных алгоритмов, Итоги науки и техн. Сер. Соврем. мат. и ее прил. Темат. обз., 2019, том 170, 38-50

DOI: https://doi.org/10.36535/0233-6723-2019-170-38-50

Использование Общероссийского математического портала Math-Net.Ru подразумевает, что вы прочитали и согласны с пользовательским соглашением

http://www.mathnet.ru/rus/agreement

Параметры загрузки:

IP : 3.89 .197 .203

26 апреля 2023 г., 08:31:13 
ИТОГИ НАУКИ И ТЕХНИКИ.

Современная математика и ее приложения.

Тематические обзоры.

Том 170 (2019). С. $38-50$

DOI: $10.36535 / 0233-6723 / 2019 / 170 / 38-50$

УДК 517.95

\title{
ОБОБЩЕННАЯ ЗАДАЧА РИМАНА О РАСПАДЕ РАЗРЫВА С ДОПОЛНИТЕЛЬНЫМИ УСЛОВИЯМИ НА ГРАНИЦЕ И ЕЕ ПРИМЕНЕНИЕ ДЛЯ ПОСТРОЕНИЯ ВЫЧИСЛИТЕЛЬНЫХ АЛГОРИТМОВ
}

() 2019 г. . И. СКАЛЬКО, С. Ю. ГРИДНЕВ

\begin{abstract}
АннотАция. Построено приближение фундаментального решения оператора задачи для гиперболической системы линейных дифференциальных уравнений первого порядка с постоянными коэффициентами. Предложен алгоритм приближенного решения обобщенной задачи Римана о распаде разрыва при наличии дополнительных условий на границах, позволяющий свести задачу нахождения значений переменных по обе стороны поверхности разрыва начальных данных к peшению системы алгебраических уравнений. Построен вычислительный алгоритм приближенного решения начально-краевой задачи для гиперболической системы линейных дифференциальных уравнений первого порядка. Алгоритм реализован для системы уравнений упругой динамики и использован для решения некоторых прикладных задач, связанных с нефтедобычей.
\end{abstract}

Ключевые слова: распад разрыва, условие сопряжения, гиперболическая система, обобщенная функция, задача Коши, матрица-функция Грина, характеристика, инвариант Римана, уравнение упругой динамики.

\author{
GENERALIZED RIEMANN PROBLEM \\ ON THE BREAKUP OF A DISCONTINUITY \\ WITH ADDITIONAL CONDITIONS AT THE BOUNDARY \\ AND ITS APPLICATION FOR CONSTRUCTING \\ COMPUTATIONAL ALGORITHMS
}

(c) 2019 YU. I. SKALKO, S. YU. GRIDNEV

\begin{abstract}
We construct an approximation of the fundamental solution of a problem for a hyperbolic system of first-order linear differential equations with constant coefficients. We propose an algorithm for an approximate solution of the generalized Riemann problem on the breakup of a discontinuity under additional conditions at the boundaries, which allows one to reduce the problem of finding the values of variables on both sides of the discontinuity surface of the initial data to the solution of a system of algebraic equations. We construct a computational algorithm for an approximate solution of the initial-boundary-value problem for a hyperbolic system of first-order linear differential equations. The algorithm is implemented for a system of equations of elastic dynamics; it is used for solving some applied problems associated with oil production.
\end{abstract}

Keywords and phrases: breakup of a discontinuity, conjugation condition, hyperbolic system, generalized function, Cauchy problem, Green matrix-function, characteristic, Riemann invariant, equation of elastic dynamics.

AMS Subject Classification: 35L40, 35L67, 35L45, 35L50 
1. Постановка задачи. Обобщенная задача Римана о распаде разрыва с дополнительными условиями на границе, которой посвящена данная работа, возникла из попыток решения конкретной прикладной задачи, которая и обусловила особенности постановки математической задачи. Поэтому сначала несколько слов о прикладной задаче.

В многочисленных промышленных экспериментах на нефтеносных месторождениях убедительно показано, что установка на поверхности виброисточника и продолжительная его работа в течение нескольких месяцев ведет к существенному повышению нефтеотдачи нефтяного пласта; в ряде случаев эффект достигает 40\%. Механизмы и процессы, ведущие к такому повышению нефтеотдачи, остаются и на сегодня неясными. В частности, не понятно, каким образом энергия упругих волн, сгенерированных виброисточником, достигает значительных глубин (1 км и более), избегая существенного рассеяния. Виброисточник с характерной мощностью 30 кВт и пятном контакта с породой порядка 1 кв.м. генерирует упругую волну. Если порода однородная, то энергия виброисточника рассеивается по полусфере и на глубинах порядка 1 км плотность энергии упругих волн убывает в $10^{6}$ раз даже при отсутствии поглощения в породе. Ожидать, что упругая волна такой малой плотности энергии вызовет какие-то значимые процессы в нефтеносном пласте, не приходится. Поскольку эффект увеличения нефтеотдачи в результате продолжительной работы поверхностного виброисточника многократно зафиксирован, то следует предположить, что при определенных условиях упругие волны распространяются в геологической породе, избегая существенного рассеяня. Мы попытались методами математического моделирования исследовать вопрос, может ли наличие трещин в геологической породе, в которых соприкасающиеся части могут смещаться друг относительно друга, приводить к тому, что упругая волна, сгенерированная виброисточником, не рассеивается по полусфере, а распространяется достаточно узким пучком так, что и на существенных глубинах ее плотность энергии остается значимой, чтобы вызвать те или иные физико-химические процессы.

Состояние геологической породы, в которой распространяется упругая волна, описывается векторным полем скорости смещений и тензором напряжений. Поскольку на трещине может происходить смещение частей породы друг относительно друга, при переходе через границу компоненты вектора скорости смещений и тензора напряжений могут претерпевать разрывы. Конечно, эти разрывы не могут быть произвольными, а обязаны удовлетворять определенным условиям, отражающим физические условия на границе соприкасающихся сред. Поскольку части геологической породы могут смещаться друг относительно друга только вдоль трещины, то компоненты вектора скорости смещений, направленные вдоль нормали к границе, должны быть непрерывными при переходе через границу. Также, в силу третьего закона Ньютона, сила, действующая со стороны одной части породы на другую, равна и противоположно направлена силе, действующей со стороны второй части породы на первую; таким образом, нормальные к границе компоненты тензора напряжений по обе стороны границы должны быть равны. Если проскальзывание происходит без трения, то тангенциальные компоненты тензора напряжений по обе стороны границы должны быть равны нулю.

С другой стороны, всюду, кроме трещины, разделяющей части породы, геологическая порода является сплошной. Следовательно, вектор смещений должен быть всюду (кроме трещины) непрерывным. Также требование выполнения третьего закона Ньютона в любом сечении ведет к непрерывности тензора напряжений. Итак, всюду, кроме трещины, и вектор смещений, и тензор напряжений непрерывны.

Исходя из сказанного задачу Римана о распаде разрыва будем рассматривать в следующей постановке. Необходимо найти решение задачи Коши для следующей системы линейных дифференциальных уравнений первого порядка с постоянными коэффициентами:

$$
\frac{\partial \boldsymbol{u}(t, \boldsymbol{x})}{\partial t}+\sum_{i=1}^{N} \boldsymbol{A}_{i} \frac{\partial \boldsymbol{u}(t, \boldsymbol{x})}{\partial x_{i}}=\mathbf{0}, \quad \boldsymbol{x} \in \mathbb{R}^{N},
$$

с начальными данными

$$
\boldsymbol{u}(t=0, \boldsymbol{x})=\boldsymbol{u}_{0}(\boldsymbol{x}),
$$


которые всюду непрерывны, кроме гиперплоскости $\Gamma: x_{1}=0$. Решение должно быть всюду непрерывным, кроме гиперплоскости Г. Также должны выполняться заданные соотношения (условия сопряжения), связывающие значения переменных по обе стороны гиперплоскости Г:

$$
\boldsymbol{L u}\left(t, x_{1}=-0, x_{2}, \ldots, x_{N}\right)+\boldsymbol{P u}\left(t, x_{1}=+0, x_{2}, \ldots, x_{N}\right)=\mathbf{0} .
$$

Сформулированную постановку будем, следуя [5], называть обобщенной задачей Римана о распаде разрыва с условиями сопряжения на границах. Обобщенная задача Римана отличается от классической задачи Римана тем, что в классической задаче начальные данные предполагаются константами по обе стороны от гиперплоскости, а в обобщенной задаче Римана начальные данные по обе стороны от гиперплоскости Г могут быть произвольными гладкими функциями, а также на всех поверхностях разрыва должны соблюдаться заданные условия сопряжения.

В случае одной пространственной переменной различными авторами предложен ряд методов решения задачи Римана (см. [5-8]). По сути, все эти методы связаны с наличием у гиперболических систем характеристик. В случае многих пространственных переменных методы, основанные на наличии характеристик, уже не работают, и задача Римана, чаще всего, решается в предположении, что вблизи разрыва решение представляет собой плоскую волну, движущуюся вдоль нормали к поверхности разрыва (см. $[5,8,9])$. Понятно, что такой подход далеко не во всех случаях является обоснованным.

В работе сформулирована постановка обобщенной задачи Римана о распаде разрыва с условиям сопряжения на границах для гиперболических систем линейных дифференциальных уравнений первого порядка с произвольным количеством пространственных переменных и приведен алгоритм построения ее решения. Этот алгоритм основан на нахождении фундаментального решения оператора задачи. Поэтому в следующих разделах будут приведены основные понятия теории обобщенных вектор-функций и построено приближение фундаментального решения оператора задачи. Построеное решение обобщенной задачи Римана ляжет в основу вычислительного алгоритма нахождения приближенного решения начально-краевой задачи для описанного класса систем дифференциальных уравнений.

2. Обобщенные вектор-функции. В дальнейшем изложении будут использоваться понятия и утверждения теории обобщенных функций, изложение которой можно найти, например в [1-4].

Определим пространство основных вектор-функций $\boldsymbol{S}\left(\mathbb{R}^{N}\right)$. Элементами этого пространства будут $M$-мерные вектор-функции $\boldsymbol{\varphi}=\left(\varphi_{1}, \ldots, \varphi_{M}\right)$, компоненты которых $\varphi_{1}(\boldsymbol{y}), \ldots, \varphi_{M}(\boldsymbol{y})$ принадлежат пространству $S\left(\mathbb{R}^{N}\right)$, которое состоит из функций класса $C^{\infty}\left(\mathbb{R}^{N}\right)$, убывающих при $|y| \rightarrow \infty$ вместе со всеми своими производными быстрее любой степени $|y|^{-1}$.

Определение 2.1. Обобщенными вектор-функциями $\boldsymbol{f}=\left(f_{1}, \ldots, f_{M}\right) \in \boldsymbol{S}^{\prime}\left(\mathbb{R}^{N}\right)$ будем называть линейные непрерывные функционалы на основном векторном пространстве $\boldsymbol{S}\left(\mathbb{R}^{N}\right)$. При этом функционал $\boldsymbol{f}$ действует на основную вектор-функцию $\varphi=\left(\varphi_{1}, \ldots, \varphi_{M}\right)$ по формуле

$$
(\boldsymbol{f}, \boldsymbol{\varphi})=\left(f_{1}, \varphi_{1}\right)+\cdots+\left(f_{M}, \varphi_{M}\right) .
$$

Определение 2.2. Обобщенным решением системы уравнений

$$
\frac{\partial \boldsymbol{u}(t, \boldsymbol{x})}{\partial t}+\sum_{i=1}^{N} \boldsymbol{A}_{i} \frac{\partial \boldsymbol{u}(t, \boldsymbol{x})}{\partial x_{i}}=\boldsymbol{f}(t, \boldsymbol{x})
$$

будем называть обобщенную функцию $\boldsymbol{u}(t, \boldsymbol{x}) \in \boldsymbol{S}^{\prime}\left(\mathbb{R}^{N+1}\right)$, удовлетворяющую этому уравнению в обобщенном смысле, т.е. для произвольной основной функции $\boldsymbol{\varphi}(t, \boldsymbol{x}) \in \boldsymbol{S}\left(\mathbb{R}^{N+1}\right)$ выполняется равенство

$$
\left(\frac{\partial \boldsymbol{u}}{\partial t}, \boldsymbol{\varphi}\right)+\sum_{i=1}^{N}\left(\boldsymbol{A}_{i} \frac{\partial \boldsymbol{u}}{\partial x_{i}}, \boldsymbol{\varphi}\right)=(\boldsymbol{f}, \boldsymbol{\varphi}),
$$

$\boldsymbol{A}_{i}$ - матрицы коэффициентов системы уравнений (4), имеющие размер $(M \times M)$. 
В дальнейшем будем полагать, что каждая из матриц $\boldsymbol{A}_{i}$ имеет полный набор левых собственных векторов и, следовательно, представима в виде

$$
\boldsymbol{A}_{i}=\boldsymbol{R}_{i} \boldsymbol{\Lambda}_{i} \boldsymbol{\Omega}_{i}
$$

$\boldsymbol{\Lambda}_{i}$ - диагональная матрица собственных чисел матрицы $\boldsymbol{A}_{i}$, упорядоченных по неубыванию, $\boldsymbol{\Omega}_{i}-$ матрица, строки которой являются левыми собственными векторами матрицы $\boldsymbol{A}_{i}$, соответствующие собственным числам $\boldsymbol{\Lambda}_{i}, \boldsymbol{R}_{i}=\boldsymbol{\Omega}_{i}^{-1}$ - матрица, столбцы которой являются правыми собственными векторами матрицы $\boldsymbol{A}_{i}$.

Определение 2.3. Фундаментальным решением оператора задачи (4) или матрицей-функцией Грина называется обобщенная матрица-функция, $\boldsymbol{G}(t, \boldsymbol{x}) \in \boldsymbol{S}^{\prime}\left(\mathbb{R}^{N+1}\right)$, удовлетворяющая уравнению

$$
\frac{\partial \boldsymbol{G}}{\partial t}+\sum_{i=1}^{N} \boldsymbol{A}_{i} \frac{\partial \boldsymbol{G}}{\partial x_{i}}=\boldsymbol{I} \delta(t, \boldsymbol{x})
$$

где $\boldsymbol{I}$ - единичная диагональная $(M \times M)$-матрица.

Определение 2.4. Сверткой $\boldsymbol{G} * \boldsymbol{f}$ обобщенной матрицы-функции $\boldsymbol{G}=G_{i j} \in \boldsymbol{S}^{\prime}$ и обобщенной вектор-функции $\boldsymbol{f}=f_{j} \in \boldsymbol{S}^{\prime}$ будем называть такую обобщенную вектор-функцию $\boldsymbol{u}=u_{i} \in \boldsymbol{S}^{\prime}$, что

$$
u_{i}=\sum_{j=1}^{M} G_{i, j} * f_{j}
$$

где $G_{i, j} * f_{j}$-свертка $G_{i, j}$ и $f_{j}$ как обобщенных функций из $S^{\prime}$.

Лемма 2.1. Пусть $\boldsymbol{f}(t, \boldsymbol{x}) \in \boldsymbol{S}^{\prime}$ такова, что свертка $\boldsymbol{G} * \boldsymbol{f}$ существует в $\boldsymbol{S}^{\prime}$. Тогда решение уравнения (4) существует в $\boldsymbol{S}^{\prime}$ и дается формулой

$$
\boldsymbol{u}=\boldsymbol{G} * \boldsymbol{f} .
$$

Это решение единственно в классе тех функиий из $\boldsymbol{S}^{\prime}$, для которых существует свертка с $\boldsymbol{G}$.

Доказательство. Пользуясь формулой дифференцирования свертки, получим

$$
\frac{\partial \boldsymbol{u}}{\partial t}+\sum_{i=1}^{N} \boldsymbol{A}_{i} \frac{\partial \boldsymbol{u}}{\partial x_{i}}=\frac{\partial(\boldsymbol{G} * \boldsymbol{f})}{\partial t}+\sum_{i=1}^{N} \boldsymbol{A}_{i} \frac{\partial(\boldsymbol{G} * \boldsymbol{f})}{\partial x_{i}}=\left(\frac{\partial \boldsymbol{G}}{\partial t}+\sum_{i=1}^{N} \boldsymbol{A}_{i} \frac{\partial \boldsymbol{G}}{\partial x_{i}}\right) * \boldsymbol{f}=\delta(t, \boldsymbol{x}) * \boldsymbol{f}=\boldsymbol{f} .
$$

Поэтому формула (7) действительно дает решение уравнения (4). Докажем единственность решения уравнения (4) в классе тех обобщенных функций из $\boldsymbol{S}^{\prime}$, для которых свертка с $\boldsymbol{G}$ существует в $\boldsymbol{S}^{\prime}$. Для этого достаточно установить, что соответствующее однородное уравнение

$$
\frac{\partial \boldsymbol{u}}{\partial t}+\sum_{i=1}^{N} \boldsymbol{A}_{i} \frac{\partial \boldsymbol{u}}{\partial x_{i}}=\mathbf{0}
$$

имеет только нулевое решение в этом классе. Но это действительно так в силу

$$
\boldsymbol{u}=\delta(t, \boldsymbol{x}) \boldsymbol{I} * \boldsymbol{u}=\left(\frac{\partial \boldsymbol{G}}{\partial t}+\sum_{i=1}^{N} \boldsymbol{A}_{i} \frac{\partial \boldsymbol{G}}{\partial x_{i}}\right) * \boldsymbol{u}=\boldsymbol{G} *\left(\frac{\partial \boldsymbol{u}}{\partial t}+\sum_{i=1}^{N} \boldsymbol{A}_{i} \frac{\partial \boldsymbol{u}}{\partial x_{i}}\right)=\mathbf{0} .
$$

Докажем две леммы, которые будут существенны в дальнейшем.

Лемма 2.2. Пусть и $(\boldsymbol{x})$-локально интегрируемая функиия в $\mathbb{R}^{N}$. Тогда

$$
\theta(t) \delta(\boldsymbol{x}-\boldsymbol{a} t) * u(\boldsymbol{x}) \delta(t)=\theta(t) u(\boldsymbol{x}-\boldsymbol{a} t) .
$$


Доказательство. Согласно определению свертки обобщенных функций (см. [1]) для произвольной основной функции $\varphi(\boldsymbol{x}, t) \in \boldsymbol{S}\left(\mathbb{R}^{N+1}\right)$ и произвольной последовательности функций $\eta_{k}(\boldsymbol{x}, \boldsymbol{y}, t, \tau) \in \boldsymbol{S}\left(\mathbb{R}^{2 N+2}\right)$, сходящейся к 1 в $\mathbb{R}^{2 N+2}$, справедлива следующая цепочка равенств:

$$
\begin{gathered}
(\theta(t) \delta(\boldsymbol{x}-\boldsymbol{a} t) * u(\boldsymbol{x}) \delta(t), \varphi(\boldsymbol{x}, t)) \stackrel{\text { def }}{=} \\
=\lim _{k \rightarrow \infty}\left(\theta(t) \delta(\boldsymbol{x}-\boldsymbol{a} t) u(\boldsymbol{y}) \delta(\tau), \eta_{k}(\boldsymbol{x}, \boldsymbol{y}, t, \tau) \varphi(\boldsymbol{x}+\boldsymbol{y}, t+\tau)\right)= \\
=\lim _{k \rightarrow \infty}\left(\theta(t) u(\boldsymbol{y}) \delta(\tau), \eta_{k}(\boldsymbol{a} t, \boldsymbol{y}, t, \tau) \varphi(\boldsymbol{a} t+\boldsymbol{y}, t+\tau)\right)= \\
=\lim _{k \rightarrow \infty} \int_{\Gamma: \tau=0} \theta(t) u(\boldsymbol{y}) \eta_{k}(\boldsymbol{a} t, \boldsymbol{y}, t, 0) \varphi(\boldsymbol{a} t+\boldsymbol{y}, t) d \Gamma=\int_{-\infty}^{+\infty} \theta(t) u(\boldsymbol{y}) \varphi(\boldsymbol{a} t+\boldsymbol{y}, t) d \boldsymbol{y} d t= \\
=\int_{-\infty}^{+\infty} \theta(t) u(\boldsymbol{x}-\boldsymbol{a} t) \varphi(\boldsymbol{x}, t) d \boldsymbol{x} d t=(\theta(t) u(\boldsymbol{x}-\boldsymbol{a} t), \varphi(\boldsymbol{x}, t)) .
\end{gathered}
$$

Замечание 2.1. Как следует из доказанной леммы, значение свертки $\theta(t) \delta(\boldsymbol{x}-\boldsymbol{a t}) * u(\boldsymbol{x}) \delta(t)$ в точке $(\boldsymbol{x}, t)$ равно значению функции $u(\boldsymbol{x})$ в точке пересечения прямой $d \boldsymbol{x} / d t=\boldsymbol{a}$, проведенной через точку $(\boldsymbol{x}, t)$, с гиперплоскостью $t=0$.

Лемма 2.3. Пусть $v(t, \boldsymbol{x})$-локально интегрируемая функиия в $\mathbb{R}^{N+1} u v(t, \boldsymbol{x})=0$ при $t \leqslant 0$. Если $a_{1} \neq 0$, mo

$$
\theta(t) \delta(\boldsymbol{x}-\boldsymbol{a} t) * v(t, \boldsymbol{x}) \delta\left(x_{1}\right)=\frac{1}{\left|a_{1}\right|} \theta\left(\frac{x_{1}}{a_{1}}\right) v\left(t-\frac{x_{1}}{a_{1}}, \boldsymbol{x}-\frac{x_{1}}{a_{1}} \boldsymbol{a}\right) ;
$$

если же $a_{1}=0$, то

$$
\theta(t) \delta(\boldsymbol{x}-\boldsymbol{a} t) * v(t, \boldsymbol{x}) \delta\left(x_{1}\right)=0 .
$$

Доказательство. Если $a_{1} \neq 0$, для произвольной основной функции $\varphi(\boldsymbol{x}, t) \in S\left(\mathbb{R}^{N+1}\right)$ и произвольной последовательности функций $\eta_{k}(\boldsymbol{x}, \boldsymbol{y}, t, \tau) \in S\left(\mathbb{R}^{2 N+2}\right)$, сходящейся к 1 в $\mathbb{R}^{2 N+2}$, справедлива следующая цепочка равенств:

$$
\begin{aligned}
& \left(\theta(t) \delta(\boldsymbol{x}-\boldsymbol{a} t) * v(t, \boldsymbol{x}) \delta\left(x_{1}\right), \varphi(\boldsymbol{x}, t)\right) \ldots \\
& \stackrel{\text { def }}{=} \lim _{k \rightarrow \infty}\left(\theta(t) \delta(\boldsymbol{x}-\boldsymbol{a} t) v(\tau, \boldsymbol{y}) \delta\left(y_{1}\right), \eta_{k}(\boldsymbol{x}, \boldsymbol{y}, t, \tau) \varphi(\boldsymbol{x}+\boldsymbol{y}, t+\tau)\right)= \\
& =\lim _{k \rightarrow \infty}\left(\theta(t) v(\tau, \boldsymbol{y}) \delta\left(y_{1}\right), \eta_{k}(\boldsymbol{a} t, \boldsymbol{y}, t, \tau) \varphi(\boldsymbol{a} t+\boldsymbol{y}, t+\tau)\right)= \\
& =\int_{\Gamma: y_{1}=0} \theta\left(t^{\prime}\right) v\left(\tau^{\prime}, \boldsymbol{y}\right) \varphi\left(\boldsymbol{a} t^{\prime}+\boldsymbol{y}, t^{\prime}+\tau^{\prime}\right) d y_{2} \ldots d y_{N} d t^{\prime} d \tau^{\prime}= \\
& =\frac{1}{\left|a_{1}\right|} \int_{-\infty}^{+\infty} \theta\left(\frac{x_{1}}{a_{1}}\right) v\left(t-\frac{x_{1}}{a_{1}}, \boldsymbol{x}-\frac{x_{1}}{a_{1}} \boldsymbol{a}\right) \varphi(\boldsymbol{x}, t) d \boldsymbol{x} d t= \\
& =\frac{1}{\left|a_{1}\right|} \theta\left(\frac{x_{1}}{a_{1}}\right) v\left(t-\frac{x_{1}}{a_{1}}, \boldsymbol{x}-\frac{x_{1}}{a_{1}} \boldsymbol{a}\right), \varphi(\boldsymbol{x}, t) .
\end{aligned}
$$

Тем самым утверждение леммы для $a_{1} \neq 0$ доказано.

Пусть $v(t, \boldsymbol{x})=0$ при $t \leqslant 0$.

Если $t>0, x_{1}<0$ и $a_{1}>0$, то $\theta(t) \delta(\boldsymbol{x}-\boldsymbol{a} t) * v(t, \boldsymbol{x}) \delta\left(x_{1}\right)=0$.

Если $t>0, x_{1}<0$ и $x_{1} / t \leqslant a_{1}<0$, то $\theta(t) \delta(\boldsymbol{x}-\boldsymbol{a t}) * v(t, \boldsymbol{x}) \delta\left(x_{1}\right)=0$.

В силу непрерывности свертки для $t>0, x_{1}<0$ имеем

$$
\theta(t) \delta\left(x_{1}\right) \delta\left(x_{2}-a_{2} t\right) \ldots \delta\left(x_{N}-a_{N} t\right) * v(t, \boldsymbol{x}) \delta\left(x_{1}\right)=\lim _{a_{1} \rightarrow 0}\left(\theta(t) \delta(\boldsymbol{x}-\boldsymbol{a} t) * v(t, \boldsymbol{x}) \delta\left(x_{1}\right)\right)=0 .
$$


Точно так же для $t>0, x_{1}>0$ имеем

$$
\theta(t) \delta\left(x_{1}\right) \delta\left(x_{2}-a_{2} t\right) \ldots \delta\left(x_{N}-a_{N} t\right) * v(t, \boldsymbol{x}) \delta\left(x_{1}\right)=\lim _{a_{1} \rightarrow 0}\left(\theta(t) \delta(\boldsymbol{x}-\boldsymbol{a} t) * v(t, \boldsymbol{x}) \delta\left(x_{1}\right)\right)=0 .
$$

Отсюда следует справедливость утверждения леммы для произвольных $\boldsymbol{a}$.

Замечание 2.2. Проведем через точку $(t, \boldsymbol{x}), t>0$, прямую линию $d \boldsymbol{x} / d t=\boldsymbol{a}$. Эта прямая пересекает гиперплоскость $x_{1}=0$ в момент времени $t^{*}=t-x_{1} / a_{1}$. Если этот момент времени лежит вне интервала $0 \leqslant t^{*} \leqslant t$, то в точке $(t, \boldsymbol{x})$

$$
\theta(t) \delta(\boldsymbol{x}-\boldsymbol{a} t) * \theta(t) v(t, \boldsymbol{x}) \delta\left(x_{1}\right)=0 .
$$

3. Фундаментальное решение. Построим фундаментальное решение оператора задачи (4). Обозначим через $\boldsymbol{V}(t, \boldsymbol{\xi})=F_{\boldsymbol{x}}[\boldsymbol{G}]$ - преобразование Фурье $\boldsymbol{G}(t, \boldsymbol{x})$ по пространственным переменным. Выполним преобразование Фурье уравнений (6) по пространственным переменным. Учитывая, что $F_{\boldsymbol{x}}[\boldsymbol{G}]=-i \xi_{j} F_{\boldsymbol{x}}[\boldsymbol{G}]$, для обобщенной функции $\boldsymbol{V}(t, \boldsymbol{\xi})$ получаем уравнение

$$
\frac{\partial \boldsymbol{V}}{\partial t}-i \sum_{j=1}^{N} \xi_{j} \boldsymbol{A}_{j} \boldsymbol{V}=\boldsymbol{I} \delta(t)
$$

Решение уравнения (8) имеет вид

$$
\boldsymbol{V}(t, \boldsymbol{\xi})=\theta(t) \exp \left(i \sum_{j=1}^{N} \xi_{j} \boldsymbol{A}_{j} t\right)
$$

где $\theta(t)$ - функция Хевисайда

$$
\theta(t)= \begin{cases}1, & \text { если } x>0, \\ 0, & \text { если } x \leqslant 0 .\end{cases}
$$

Следуя определению матричной экспоненты,

$$
\exp \left(i \sum_{j=1}^{N} \xi_{j} \boldsymbol{A}_{j} t\right)=\prod_{j=1}^{N} \exp \left(i \xi_{j} \boldsymbol{A}_{j} t\right)+\sum_{|\boldsymbol{\alpha}| \geqslant 2} t^{|\boldsymbol{\alpha}|} \boldsymbol{B}_{\boldsymbol{\alpha}} \prod_{j=1}^{N}\left(-i \xi_{j}\right)^{\alpha_{j}} .
$$

Здесь $\boldsymbol{\alpha}=\left(\alpha_{1}, \alpha_{2}, \ldots, \alpha_{N}\right)$ - целочисленный вектор с неотрицательными составляющими $\alpha_{j}$ (мультииндекс), $|\boldsymbol{\alpha}|=\alpha_{1}+\cdots+\alpha_{N}, \boldsymbol{B}_{\boldsymbol{\alpha}}$ - матрицы размера $M \times M$, являющиеся полиномами матриц $\boldsymbol{A}_{j}$ степени $|\boldsymbol{\alpha}|$.

Учтем (5); тогда

$$
\exp \left(i \xi_{j} \boldsymbol{A}_{j} t\right)=\boldsymbol{R}_{j} \exp \left(i \xi_{j} \boldsymbol{\Lambda}_{j} t\right) \boldsymbol{\Omega}_{j}
$$

Следовательно,

$$
\exp \left(i \sum_{j=1}^{N} \xi_{j} \boldsymbol{A}_{j} t\right)=\prod_{j=1}^{N} \boldsymbol{R}_{j} \exp \left(i \xi_{j} \boldsymbol{\Lambda}_{j} t\right) \boldsymbol{\Omega}_{j}+\sum_{|\boldsymbol{\alpha}| \geqslant 2} t^{|\boldsymbol{\alpha}|} \boldsymbol{B}_{\boldsymbol{\alpha}} \prod_{j=1}^{N}\left(-i \xi_{j}\right)^{\alpha_{j}} .
$$

Выполняя обратное преобразование Фурье, получим матрицу-функцию Грина

$$
\boldsymbol{G}(t, \boldsymbol{x})=\theta(t)\left(\prod_{j=1}^{N} \boldsymbol{R}_{j} \delta\left(\boldsymbol{I} x_{j}-\boldsymbol{\Lambda}_{j} t\right) \boldsymbol{\Omega}_{j}+\sum_{|\boldsymbol{\alpha}| \geqslant 2} t^{|\boldsymbol{\alpha}|} \boldsymbol{B}_{\boldsymbol{\alpha}} D^{\boldsymbol{\alpha}} \delta(\boldsymbol{x})\right) ;
$$

здесь $\delta\left(\boldsymbol{I} x_{j}-\boldsymbol{\Lambda}_{j} t\right)$ - диагональные матрицы, в $k$-й строке которых стоит обобщенная функция $\delta\left(x_{j}-\lambda_{j}^{k} t\right), \lambda_{j}^{k}-k$-е собственное число матрицы $\boldsymbol{A}_{j}, D^{\boldsymbol{\alpha}}=\frac{\partial^{|\boldsymbol{\alpha}|}}{\partial x_{1}^{\alpha_{1}} \partial x_{2}^{\alpha_{2}} \ldots \partial x_{N}^{\alpha_{N}}}$-оператор дифференцирования по пространственным переменным. 
Рассмотрим сомножитель $\boldsymbol{R}_{j} \delta\left(\boldsymbol{I} x_{j}-\boldsymbol{\Lambda}_{j} t\right) \boldsymbol{\Omega}_{j}$. Обозначим через $\boldsymbol{D}^{k}$ квадратную матрицу размера $M \times M$, все элементы которой равны 0 , кроме $k$-го элемента главной диагонали, равного 1 . Тогда

$$
\boldsymbol{R}_{j} \delta\left(\boldsymbol{I} x_{j}-\boldsymbol{\Lambda}_{j} t\right) \boldsymbol{\Omega}_{j}=\sum_{k=1}^{M} \boldsymbol{R}_{j} \boldsymbol{D}^{k} \boldsymbol{\Omega}_{j} \delta\left(x_{j}-\lambda_{j}^{k} t\right)=\sum_{k=1}^{M} \boldsymbol{C}_{j}^{k} \delta\left(x_{j}-\lambda_{j}^{k} t\right) .
$$

Следовательно,

$$
\prod_{j=1}^{N} \boldsymbol{R}_{j} \delta\left(\boldsymbol{I} x_{j}-\boldsymbol{\Lambda}_{j} t\right) \boldsymbol{\Omega}_{j}=\sum_{k_{1}=1}^{M} \sum_{k_{2}=1}^{M} \ldots \sum_{k_{N}=1}^{M} \boldsymbol{C}_{1}^{k_{1}} \boldsymbol{C}_{2}^{k_{2}} \ldots \boldsymbol{C}_{N}^{k_{N}} \delta\left(x_{1}-\lambda_{1}^{k_{1}} t\right) \delta\left(x_{2}-\lambda_{2}^{k_{2}} t\right) \ldots \delta\left(x_{N}-\lambda_{N}^{k_{N}} t\right) .
$$

Введем мультииндекс $\boldsymbol{k}=\left(k_{1}, k_{2}, \ldots, k_{N}\right)$ с целочисленными составляющими $k_{j}=1, \ldots, M$, многоиндексный массив матриц $C^{k}=C_{1}^{k_{1}} C_{2}^{k_{2}} \ldots C_{N}^{k_{N}}$, многоиндексный массив векторов $\lambda^{k}=\left(\lambda_{1}^{k_{1}}, \lambda_{2}^{k_{2}}, \ldots, \lambda_{N}^{k_{N}}\right)$, то

$$
\prod_{j=1}^{N} \boldsymbol{R}_{j} \delta\left(\boldsymbol{I} x_{j}-\boldsymbol{\Lambda}_{j} t\right) \boldsymbol{\Omega}_{j}=\sum_{\boldsymbol{k}} \boldsymbol{C}^{\boldsymbol{k}} \delta\left(\boldsymbol{x}-\boldsymbol{\lambda}^{\boldsymbol{k}} t\right)
$$

тогда

$$
\boldsymbol{G}(t, \boldsymbol{x})=\theta(t) \sum_{\boldsymbol{k}} \boldsymbol{C}^{\boldsymbol{k}} \delta\left(\boldsymbol{x}-\boldsymbol{\lambda}^{\boldsymbol{k}} t\right)+O\left(t^{2}\right)
$$

В случае двух пространственных переменых

$$
\boldsymbol{G}(t, \boldsymbol{x})=\theta(t) \sum_{\boldsymbol{k}} \boldsymbol{C}_{1}^{k_{1}} \boldsymbol{C}_{2}^{k_{2}} \delta\left(\boldsymbol{x}-\boldsymbol{\lambda}^{\boldsymbol{k}} t\right)+\frac{\theta(t)}{2} t^{2}\left(\boldsymbol{A}_{2} \boldsymbol{A}_{1}-\boldsymbol{A}_{1} \boldsymbol{A}_{2}\right) \frac{\partial^{2} \delta(\boldsymbol{x})}{\partial x_{1} \partial x_{2}}+O\left(t^{3}\right) .
$$

Если поменять нумерацию пространственных переменных, то можем записать

$$
\boldsymbol{G}(t, \boldsymbol{x})=\theta(t) \sum_{\boldsymbol{k}} \boldsymbol{C}_{2}^{k_{2}} \boldsymbol{C}_{1}^{k_{1}} \delta\left(\boldsymbol{x}-\boldsymbol{\lambda}^{\boldsymbol{k}} t\right)-\frac{\theta(t)}{2} t^{2}\left(\boldsymbol{A}_{2} \boldsymbol{A}_{1}-\boldsymbol{A}_{1} \boldsymbol{A}_{2}\right) \frac{\partial^{2} \delta(\boldsymbol{x})}{\partial x_{1} \partial x_{2}}+O\left(t^{3}\right) .
$$

Сопоставляя (9) и (10), получаем

$$
\boldsymbol{G}(t, \boldsymbol{x})=\theta(t) \sum_{\boldsymbol{k}} \overline{\boldsymbol{C}}^{\boldsymbol{k}} \delta\left(\boldsymbol{x}-\boldsymbol{\lambda}^{\boldsymbol{k}} t\right)+O\left(t^{3}\right) .
$$

Здесь использовано обозначение

$$
\overline{\boldsymbol{C}}^{\boldsymbol{k}}=\frac{1}{2}\left(\boldsymbol{C}_{1}^{k_{1}} \boldsymbol{C}_{2}^{k_{2}}+\boldsymbol{C}_{2}^{k_{2}} \boldsymbol{C}_{1}^{k_{1}}\right) .
$$

Обратим внимание на следующий факт, который будет использован в дальнейшем. Поскольку $\boldsymbol{C}^{k_{j}}=\boldsymbol{R}_{j} \boldsymbol{D}^{k_{j}} \boldsymbol{\Omega}_{j}$, то

$$
\sum_{k_{j}=1}^{M} \boldsymbol{C}^{k_{j}}=\boldsymbol{R}_{j}\left(\sum_{k_{j}=1}^{M} \boldsymbol{D}^{k_{j}}\right) \boldsymbol{\Omega}_{j}=\boldsymbol{R}_{j} \boldsymbol{I} \boldsymbol{\Omega}_{j}=\boldsymbol{I} .
$$

4. Задача Римана. Пусть $\boldsymbol{u}(t, \boldsymbol{x})$ - решение задачи Римана (1)-(3). Введем обозначение

$$
\boldsymbol{v}(t, \boldsymbol{x})=\theta(t)\left(\boldsymbol{u}\left(t, x_{1}=+0, x_{2}, \ldots, x_{N}\right)-\boldsymbol{u}\left(t, x_{1}=-0, x_{2}, \ldots, x_{N}\right)\right) .
$$

Покажем, что функция $\boldsymbol{u}(t, \boldsymbol{x})$, рассматриваемая как обобщенная функция из $\boldsymbol{S}^{\prime}$, удовлетворяет уравнению

$$
\frac{\partial \boldsymbol{u}}{\partial t}+\sum_{i=1}^{N} \boldsymbol{A}_{i} \frac{\partial \boldsymbol{u}}{\partial x_{i}}=\boldsymbol{u}_{0} \delta(t)+\boldsymbol{A}_{1} \boldsymbol{v} \delta\left(x_{1}\right) .
$$


Действительно, при всех $\boldsymbol{\varphi}(t, \boldsymbol{x}) \in \boldsymbol{S}$ имеем цепочку равенств

$$
\begin{gathered}
\left(\frac{\partial \boldsymbol{u}}{\partial t}+\sum_{i=1}^{N} \boldsymbol{A}_{i} \frac{\partial \boldsymbol{u}}{\partial x_{i}}, \boldsymbol{\varphi}\right)=-\int\left(\frac{\partial \boldsymbol{\varphi}^{T}}{\partial t} \boldsymbol{u}+\sum_{i=1}^{N} \frac{\partial \boldsymbol{\varphi}^{T}}{\partial x_{i}} \boldsymbol{A}_{i} \boldsymbol{u}\right) d t d \boldsymbol{x}=\cdots= \\
=\int\left(\boldsymbol{\varphi}^{T}\left(\frac{\partial \boldsymbol{u}}{\partial t}+\sum_{i=1}^{N} \boldsymbol{A}_{i} \frac{\partial \boldsymbol{u}}{\partial x_{i}}\right)\right) d t d \boldsymbol{x}+\int\left(\boldsymbol{\varphi}^{T}(0, \boldsymbol{x}) \boldsymbol{u}(0, \boldsymbol{x})\right) d \boldsymbol{x}+\int_{\Gamma}\left(\boldsymbol{\varphi}^{T} \boldsymbol{A}_{1} \boldsymbol{v}\right) d t d \Gamma
\end{gathered}
$$

откуда и вытекает равенство (12).

Решение уравнения (12) представимо в виде свертки

$$
\boldsymbol{u}=\boldsymbol{G} * \boldsymbol{u}_{0} \delta(t)+\boldsymbol{G} * \boldsymbol{A}_{1} \boldsymbol{v} \delta\left(x_{1}\right) .
$$

На основании лемм 2.2 и 2.3 для точек $\boldsymbol{x}=\left(x_{1}, x_{2}, \ldots, x_{N}\right)$, лежащих в левой полуплоскости $\left(x_{1} \leqslant 0\right)$, с точностью до $O\left(t^{2}\right)$ можем записать

$$
\boldsymbol{u}\left(t, x_{1}, x_{2}, \ldots, x_{N}\right)=\sum_{\boldsymbol{k}} \boldsymbol{C}^{\boldsymbol{k}} \boldsymbol{u}_{0}\left(\boldsymbol{x}-\boldsymbol{\lambda}^{\boldsymbol{k}} t\right)-\sum_{\boldsymbol{k}: x_{1}>\lambda_{-}^{k_{1}} t} \frac{\boldsymbol{C}^{\boldsymbol{k}} \boldsymbol{A}_{1}}{\lambda^{k_{1}}} \boldsymbol{v}\left(t-\frac{x_{1}}{\lambda^{k_{1}}}, \boldsymbol{x}-\frac{x_{1}}{\lambda^{k_{1}}} \boldsymbol{\lambda}^{\boldsymbol{k}}\right) .
$$

Переходя в $(13)$ к пределу при $x_{1} \rightarrow-0$, получаем

$$
\boldsymbol{u}\left(t, x_{1}=-0, x_{2}, \ldots, x_{N}\right)=\sum_{\boldsymbol{k}} \boldsymbol{C}^{\boldsymbol{k}} \boldsymbol{u}_{0}\left(-\boldsymbol{\lambda}^{k_{1}} t, x_{2}-\boldsymbol{\lambda}^{k_{2}} t, \ldots, x_{N}-\boldsymbol{\lambda}^{k_{N}} t\right)-\sum_{\boldsymbol{k}: \lambda^{k_{1}}<0} \frac{\boldsymbol{C}^{\boldsymbol{k}} \boldsymbol{A}_{1}}{\lambda^{k_{1}}} \boldsymbol{v} .
$$

Учитывая (11), имеем

$$
\sum_{\boldsymbol{k}: \lambda^{k_{1}}<0} \frac{1}{\lambda^{k_{1}}} \boldsymbol{C}^{\boldsymbol{k}} \boldsymbol{A}_{1}=\sum_{k_{1}: \lambda^{k_{1}}<0} \frac{1}{\lambda^{k_{1}}} \boldsymbol{C}^{k_{1}} \boldsymbol{A}_{1} .
$$

Поскольку $\boldsymbol{A}_{1}=\boldsymbol{R}_{1} \boldsymbol{\Lambda}_{1} \boldsymbol{\Omega}_{1}$ и $\boldsymbol{C}^{k_{1}}=\boldsymbol{R}_{1} \boldsymbol{D}^{k_{1}} \boldsymbol{\Omega}_{1}$, то $\left(1 / \lambda^{k_{1}}\right) \boldsymbol{C}^{k_{1}} \boldsymbol{A}_{1}=\boldsymbol{C}^{k_{1}}$. Равенство (14) принимает вид

$$
\boldsymbol{u}\left(t, x_{1}=-0, x_{2}, \ldots, x_{N}\right)=\sum_{\boldsymbol{k}} \boldsymbol{C}^{\boldsymbol{k}} \boldsymbol{u}_{0}\left(-\boldsymbol{\lambda}^{k_{1}} t, x_{2}-\boldsymbol{\lambda}^{k_{2}} t, \ldots, x_{N}-\boldsymbol{\lambda}^{k_{N}} t\right)-\sum_{k_{1}: \lambda^{k_{1}}<0} \boldsymbol{C}^{k_{1}} \boldsymbol{v}
$$

Аналогично, для точек $\boldsymbol{x}=\left(x_{1}, x_{2}, \ldots, x_{N}\right)$, лежащих в правой полуплоскости $\left(x_{1} \geqslant 0\right)$, с точностью до $O\left(t^{2}\right)$ можем записать

$$
\boldsymbol{u}\left(t, x_{1}, x_{2}, \ldots, x_{N}\right)=\sum_{\boldsymbol{k}} \boldsymbol{C}^{\boldsymbol{k}} \boldsymbol{u}_{0}\left(\boldsymbol{x}-\boldsymbol{\lambda}^{\boldsymbol{k}} t\right)+\sum_{\boldsymbol{k}: x_{1}<\lambda_{-}^{k_{1}} t} \frac{\boldsymbol{C}^{\boldsymbol{k}} \boldsymbol{A}_{1}}{\lambda^{k_{1}}} \boldsymbol{v}\left(t-\frac{x_{1}}{\lambda^{k_{1}}}, \boldsymbol{x}-\frac{x_{1}}{\lambda^{k_{1}}} \boldsymbol{\lambda}^{\boldsymbol{k}}\right) .
$$

Переходя в (16) к пределу при $x_{1} \rightarrow+0$, аналогично (15) получаем

$$
\boldsymbol{u}\left(t, x_{1}=+0, x_{2}, \ldots, x_{N}\right)=\sum_{\boldsymbol{k}} \boldsymbol{C}^{\boldsymbol{k}} \boldsymbol{u}_{0}\left(-\boldsymbol{\lambda}^{k_{1}} t, x_{2}-\boldsymbol{\lambda}^{k_{2}} t, \ldots, x_{N}-\boldsymbol{\lambda}^{k_{N}} t\right)+\sum_{k_{1}: \lambda^{k_{1}}>0} \boldsymbol{C}^{k_{1}} \boldsymbol{v}
$$

Подставим выражения (15) и (17) в условия сопряжения (3). Получим систему уравнений, которым должен удовлетворять скачок переменных $\boldsymbol{v}(t, \boldsymbol{x})$ при переходе через гиперплоскость $\Gamma$, заданную уравнением $x_{1}=0$ :

$$
\sum_{k_{1}: \lambda^{k_{1}}>0} \boldsymbol{P} \boldsymbol{C}^{k_{1}} \boldsymbol{v}-\sum_{k_{1}: \lambda^{k_{1}}<0} \boldsymbol{L} \boldsymbol{C}^{k_{1}} \boldsymbol{v}=\boldsymbol{b}^{\boldsymbol{k}} .
$$

Здесь

$$
\begin{aligned}
\boldsymbol{b}^{\boldsymbol{k}}=-\sum_{\boldsymbol{k}} \boldsymbol{P} \boldsymbol{C}^{\boldsymbol{k}} \boldsymbol{u}_{0}\left(+0-\boldsymbol{\lambda}^{k_{1}} t, x_{2}-\boldsymbol{\lambda}^{k_{2}} t, \ldots, x_{N}-\boldsymbol{\lambda}^{k_{N}} t\right)- \\
\quad-\sum_{\boldsymbol{k}} \boldsymbol{L} \boldsymbol{C}^{\boldsymbol{k}} \boldsymbol{u}_{0}\left(-0-\boldsymbol{\lambda}^{k_{1}} t, x_{2}-\boldsymbol{\lambda}^{k_{2}} t, \ldots, x_{N}-\boldsymbol{\lambda}^{k_{N}} t\right) .
\end{aligned}
$$


Вычтя из уравнения (17) уравнение (15), получим систему уравнений, которым также должен удовлетворять скачок значений переменных $\boldsymbol{v}(t, \boldsymbol{x})$ :

$$
\sum_{k_{1}: \lambda^{k_{1}}=0} \boldsymbol{C}^{k_{1}} \boldsymbol{v}=\sum_{\boldsymbol{k}: \lambda^{k_{1}}=0} \boldsymbol{C}^{\boldsymbol{k}} \boldsymbol{d}^{\boldsymbol{k}}
$$

Здесь

$$
\boldsymbol{d}^{\boldsymbol{k}}=\boldsymbol{u}_{0}\left(+0, x_{2}-\boldsymbol{\lambda}^{k_{2}} t, \ldots, x_{N}-\boldsymbol{\lambda}^{k_{N}} t\right)-\boldsymbol{u}_{0}\left(-0, x_{2}-\boldsymbol{\lambda}^{k_{2}} t, \ldots, x_{N}-\boldsymbol{\lambda}^{k_{N}} t\right) .
$$

Количество линейно независимых уравнений системы (19) равно кратности нулевого собственного числа матрицы $\boldsymbol{A}_{1}$. Умножим равенство (19) на левые собственные векторы-строки матрицы $\boldsymbol{A}_{1}$, соответствующие нулевому собственному числу:

$$
\boldsymbol{l}^{k_{1}} \boldsymbol{v}=\sum_{\boldsymbol{k}: \lambda^{k_{1}}=0} \boldsymbol{l}^{k_{1}} \boldsymbol{C}^{\boldsymbol{k}} \boldsymbol{d}^{\boldsymbol{k}}, \quad k_{1}: \lambda^{k_{1}}=0
$$

Объединив равенства (18) и (20), получим систему линейных алгебраических уравнений, которым должен удовлетворять скачок переменных $\boldsymbol{v}(t, \boldsymbol{x})$ при переходе через гиперплоскость $\Gamma: x_{1}=0$ :

$$
\left\{\begin{array}{c}
\sum_{k_{1}: \lambda^{k_{1}}>0} \boldsymbol{P} \boldsymbol{C}^{k_{1}} \boldsymbol{v}-\sum_{k_{1}: \lambda^{k_{1}}<0} \boldsymbol{L} \boldsymbol{C}^{k_{1}} \boldsymbol{v}=\boldsymbol{b}^{\boldsymbol{k}}, \\
\boldsymbol{l}^{k_{1}} \boldsymbol{v}=\sum_{\boldsymbol{k}: \lambda^{k_{1}}=0} \boldsymbol{l}^{k_{1}} \boldsymbol{C}^{\boldsymbol{k}} \boldsymbol{d}^{\boldsymbol{k}}, \quad k_{1}: \lambda^{k_{1}}=0 .
\end{array}\right.
$$

Для однозначного решения обобщенной задачи Римана о распаде разрыва с условиями сопряжения на границе необходимо и достаточно, чтобы система уравнений (21) имела единственное решение. Решая систему уравнений $(21)$, определим значение $\boldsymbol{v}(t, \boldsymbol{x})$.

Формулы (13) и (16) с полученными зависимостями $\boldsymbol{v}(t, \boldsymbol{x})$ дают полное решение обобщенной задачи Римана о распаде разрыва для случая многих пространственных переменных.

Построенное приближение решения обобщенной задачи Римана с дополнительными условиями сопряжения на разрыве является точным в случае одной пространственной переменой. Также это решение будет точным и в случае многих пространственных переменных, если начальные данные заданы линейными функциями по обе стороны гиперплоскости $\Gamma: x_{1}=0$.

5. Граничные условия. Рассмотрим еще одну задачу, которая далее будет использована при построении вычислительного алгоритма. Необходимо найти в полупространстве $x_{1} \leqslant 0$ решение начально-краевой задачи для системы линейных дифференциальных уравнений первого порядка с постоянными коэффициентами (1) с начальными данными (2). Решение должно быть непрерывным в полупространстве $x_{1} \leqslant 0$ и удовлетворять заданным граничным условиям на гиперплоскости $\Gamma: x_{1}=0$ :

$$
\boldsymbol{L u}\left(t, x_{1}=-0, x_{2}, \ldots, x_{N}\right)=\mathbf{0} .
$$

Полагаем, что начальные данные удовлетворяют граничным условиям. Эту задачу будем называть обобщенной задачей Римана с граничными условиями.

Пусть $\boldsymbol{u}(t, \boldsymbol{x})$ - решение этой задачи. Доопределим $\boldsymbol{u}(t, \boldsymbol{x})$ нулем для $t<0$ и $t \geqslant 0, x_{1}>$ 0 . Также доопределим нулем $\boldsymbol{u}_{0}(\boldsymbol{x})$ для $x_{1}>0$. Введем обозначение $\boldsymbol{v}(t, \boldsymbol{x})=-\theta(t) \boldsymbol{u}\left(t, x_{1}=\right.$ $\left.-0, x_{2}, \ldots, x_{N}\right)$.

Как было показано выше, функция $\boldsymbol{u}(t, \boldsymbol{x})$, рассматриваемая как обобщенная функция из $\boldsymbol{S}^{\prime}$, удовлетворяет уравнению (12). Решение этого уравнения задается формулой (13). Вектор $\boldsymbol{v}(t, \boldsymbol{x})$ с точностью $O\left(t^{2}\right)$ удовлетворяет равенствам (15), которые можно переписать в виде

$$
-\sum_{k_{1}: \lambda^{k_{1}} \geqslant 0} \boldsymbol{C}^{k_{1}} \boldsymbol{v}=\sum_{\boldsymbol{k}: \lambda^{k_{1}} \geqslant 0} \boldsymbol{C}^{\boldsymbol{k}} \boldsymbol{u}_{0}\left(-\boldsymbol{\lambda}^{k_{1}} t, x_{2}-\boldsymbol{\lambda}^{k_{2}} t, \ldots, x_{N}-\boldsymbol{\lambda}^{k_{N}} t\right) .
$$


Количество линейно независимых уравнений системы (23) равно количеству линейно независимых собственных векторов матрицы $\boldsymbol{A}_{1}$, соответствующих неотрицательным собственным числам. Умножим равенство (23) на левые собственные вектора-строки матрицы $\boldsymbol{A}_{1}$, соответствующие неотрицательным собственным числам

$$
-\boldsymbol{l}^{k_{1}} \boldsymbol{v}=\sum_{\boldsymbol{k}: \lambda^{k_{1}} \geqslant 0} \boldsymbol{l}^{k_{1}} \boldsymbol{C}^{\boldsymbol{k}} \boldsymbol{u}_{0}\left(-\boldsymbol{\lambda}^{k_{1}} t, x_{2}-\boldsymbol{\lambda}^{k_{2}} t, \ldots, x_{N}-\boldsymbol{\lambda}^{k_{N}} t\right), \quad k_{1}: \lambda^{k_{1}} \geqslant 0 .
$$

Объединим равенства (24) и (22), получим систему линейных алгебраических уравнений, которым должны удовлетворять значения решения задачи на границе $\Gamma$ :

$$
\left\{\begin{aligned}
-\boldsymbol{l}^{k_{1}} \boldsymbol{v} & =\sum_{\boldsymbol{k}: \lambda^{k_{1}} \geqslant 0} \boldsymbol{l}^{k_{1}} \boldsymbol{C}^{\boldsymbol{k}} \boldsymbol{u}_{0}\left(-\boldsymbol{\lambda}^{k_{1}} t, x_{2}-\boldsymbol{\lambda}^{k_{2}} t, \ldots, x_{N}-\boldsymbol{\lambda}^{k_{N}} t\right), \quad k_{1}: \lambda^{k_{1}} \geqslant 0, \\
\boldsymbol{L} \boldsymbol{v} & =\mathbf{0}
\end{aligned}\right.
$$

Решим систему уравнений (25) и определим значение $\boldsymbol{v}(t, \boldsymbol{x})$ при $t>0$ на гиперплоскости $x_{1}=0$. Формулы (13) с полученными зависимостями $\boldsymbol{v}(t, \boldsymbol{x})$ дают полное решение обобщенной задачи Римана с граничными условиями для случая многих пространственных переменных с точностью до $O\left(t^{2}\right)$. Опять же, если начальные данные $\boldsymbol{u}_{0}(\boldsymbol{x})$ являются линейными функциями, то полученное решение является точным решением этой задачи.

В частности, если граничные условия имеют вид

$$
\sum_{k: \lambda_{k_{1}}^{-}<0} C^{\boldsymbol{k}} \boldsymbol{v}=\mathbf{0}
$$

что означает, что любые волны проходят через границу, не отражаясь, то такие условия называются «прозрачными». В этом случае, если умножить уравнения граничных условий слева на левые собственные вектора-строки матрицы $\boldsymbol{A}_{1}$, то система уравнений (25) принимает вид

$$
\left\{\begin{aligned}
-\boldsymbol{l}^{k_{1}} \boldsymbol{v} & =\sum_{\boldsymbol{k}: \lambda^{k_{1}} \geqslant 0} \boldsymbol{l}^{k_{1}} \boldsymbol{C}^{\boldsymbol{k}} \boldsymbol{u}_{0}\left(-\boldsymbol{\lambda}^{k_{1}} t, x_{2}-\boldsymbol{\lambda}^{k_{2}} t, \ldots, x_{N}-\boldsymbol{\lambda}^{k_{N}} t\right), & & k_{1}: \lambda^{k_{1}} \geqslant 0, \\
\boldsymbol{l}^{k_{1}} \boldsymbol{v} & =\mathbf{0}, & & k_{1}: \lambda^{k_{1}}<0 .
\end{aligned}\right.
$$

Поскольку система уравнений (1) гиперболична и матрица $\boldsymbol{A}_{1}$ имеет полный набор линейно независимых левых собственных векторов, система уравнений (26) совместна и обобщенная задача Римана с прозрачными граничными условиями имеет единственное решение.

6. Распространения упругих волн в блочно-трещиноватой среде. Построение вычислительного алгоритма, основанного на приведенных выше результатах, продемонстрируем на задаче распространения упругих волн в неоднородной блочно-трещиноватой среде.

6.1. Математическая модель. Систему уравнений, описывающую распространение упругих волн, для случая двух пространственных переменных, следуя [8], можно записать в виде:

$$
\begin{aligned}
\frac{\partial}{\partial t} \sigma_{11}-(\lambda+2 \mu) \frac{\partial}{\partial x_{1}} v_{1}-\lambda \frac{\partial}{\partial x_{2}} v_{2} & =0 \\
\frac{\partial}{\partial t} \sigma_{22}-\lambda \frac{\partial}{\partial x_{1}} v_{1}-(\lambda+2 \mu) \frac{\partial}{\partial x_{2}} v_{2} & =0 \\
\frac{\partial}{\partial t} \sigma_{12}-\mu \frac{\partial}{\partial x_{1}} v_{2}-\mu \frac{\partial}{\partial x_{2}} v_{1} & =0 \\
\rho \frac{\partial}{\partial t} v_{1}-\frac{\partial}{\partial x_{1}} \sigma_{11}-\frac{\partial}{\partial x_{2}} \sigma_{12} & =0 \\
\rho \frac{\partial}{\partial t} v_{2}-\frac{\partial}{\partial x_{1}} \sigma_{12}-\frac{\partial}{\partial x_{2}} \sigma_{22} & =0
\end{aligned}
$$


где $\lambda$ и $\mu$-коэффициенты Ламе, $\rho$-массовая плотность среды, $\sigma_{11}, \sigma_{22}, \sigma_{12}$ - компоненты тензора напряжений, $v_{1}, v_{2}$-компоненты вектора скорости смещений. Введя вектор переменных $\boldsymbol{u}=\left(\sigma_{11}, \sigma_{22}, \sigma_{12}, v_{1}, v_{2}\right)^{T}$ и матрицы

$$
\boldsymbol{A}_{1}=\left(\begin{array}{ccccc}
0 & 0 & 0 & -(\lambda+2 \mu) & 0 \\
0 & 0 & 0 & -\lambda & 0 \\
0 & 0 & 0 & 0 & -\mu \\
-1 / \rho & 0 & 0 & 0 & 0 \\
0 & 0 & -1 / \rho & 0 & 0
\end{array}\right), \quad \boldsymbol{A}_{2}=\left(\begin{array}{ccccc}
0 & 0 & 0 & 0 & -\lambda \\
0 & 0 & 0 & 0 & -(\lambda+2 \mu) \\
0 & 0 & 0 & -\mu & 0 \\
0 & 0 & 0 & -1 / \rho & 0 \\
0 & -1 / \rho & 0 & 0 & 0
\end{array}\right)
$$

эту систему уравнений можно записать в виде (1).

Система уравнений (27) является гиперболической, матрицы $\boldsymbol{A}_{1}$ и $\boldsymbol{A}_{2}$ имеют полный набор линейно независимых собственных векторов и предствимы в виде (5).

Далее все размерные величины заданы в системе единиц СИ. Поставим для этой системы уравнений следующую задачу. В области

$$
\Omega=\left[-30<x_{1}<30,-600<x_{2}<0\right]
$$

необходимо найти решение начально-краевой задачи для системы уравнений (27). Уравнения (27) должны выполняться всюду в $\Omega$, кроме внутренних границ $\Gamma_{\gamma}, \gamma=1,2$, задаваемых условием $\Gamma_{1}: x_{1}=-15$ и $\Gamma_{2}: x_{1}=15$. На этих границах должны выполняться «условия проскальзывания без трения», состоящие в том, что при переходе через эти границы непрерывны нормальные к границам компоненты вектора смещений, нормальные к границе компоненты силы с разных сторон границы равны по величине и противоположно направлены, тангенциальные компоненты сил, действующих по обе стороны границы, равны 0 :

$$
\begin{aligned}
v_{1}\left(t, \boldsymbol{x} \in \Gamma_{\gamma}^{-}\right)-v_{1}\left(t, \boldsymbol{x} \in \Gamma_{\gamma}^{+}\right) & =0, \\
\sigma_{11}\left(t, \boldsymbol{x} \in \Gamma_{\gamma}^{-}\right)-\sigma_{11}\left(t, \boldsymbol{x} \in \Gamma_{\gamma}^{+}\right) & =0, \\
\sigma_{12}\left(t, \boldsymbol{x} \in \Gamma_{\gamma}^{-}\right) & =0 \\
\sigma_{12}\left(t, \boldsymbol{x} \in \Gamma_{\gamma}^{+}\right) & =0 .
\end{aligned}
$$

На внешних границах $x_{1}=-30, x_{2}=-600, x_{1}=30$, должны выполняться прозрачные граничные условия.

На границе $x_{2}=0$ работает источник вибровоздействия, который действует на геологическую среду с усилием $F_{j} \sin \omega t, j=1,2$. Это усилие распределено по границе с плотностью $P_{j}\left(x_{1}\right) \sin \omega t$, так что

$$
\int P_{j} d x_{1}=F_{j}
$$

В соответствии с третьим законом Ньютона во всех точках этой границы должны выполняться условия

$$
\sigma_{12}=-P_{1} \sin \omega t, \quad \sigma_{22}=-P_{2} \sin \omega t
$$

6.2. Численныц алгоритм. Внутренними границами область $\Omega$ разбивается на подобласти

$$
\begin{aligned}
& \Omega_{1}=\left[-30<x_{1}<-15,-600<x_{2}<0\right], \\
& \Omega_{2}=\left[-15<x_{1}<15,-600<x_{2}<0\right], \\
& \Omega_{3}=\left[15<x_{1}<30,-600<x_{2}<0\right] .
\end{aligned}
$$

Построим в каждой из подобластей прямоугольную сетку со сторонами параллельными осям координат, так, чтобы узлы, лежащие на внутренних границах совпадали для обеих прилегающих подобластей. Сетку строим равномерной по каждой из координат и $h_{j}, j=1,2$, шаг сетки по соответствующим направлениям. Пусть индекс $p_{1}$ нумерует узлы сетки в первой подобласти $\Omega_{1}$, индекс $p_{2}$ нумерует узлы во второй подобласти $\Omega_{2}$ и индекс $p_{3}$ нумерует узлы сетки в третьей подобласти $\Omega_{3}$. Далее для нумерации узлов сетки в подобласти будем использовать, там где это 
не вызовет недоразумения, индекс $p$, каждый раз подразумевая, что в каждой подобласти этот индекс пробегает свой набор значений.

Будем полагать, что усилие, создаваемое виброисточником, направлено по вертикали, т.е. $P_{1}\left(x_{1}\right)=0$, а распределение $P_{2}\left(x_{1}\right)$ является кусочно линейной функцией, равной нулю во всех узлах границы $x_{2}=0$, кроме узла с координатами $x_{1}=0, x_{2}=0$. В этом узле $P_{1}$ принимает значение $F_{2} / h_{1}$.

Определим равномерную сетку по времени $t_{m}=m \tau, m=0: 1: M$. Шаг сетки $\tau$ должен удовлетворять условию

$$
\tau \leqslant \min \left(\min _{k} \frac{h_{1}}{\lambda_{1}^{k}}, \min _{k} \frac{h_{2}}{\lambda_{2}^{k}}\right) .
$$

В каждой подобласти построим систему базисных полиномов $H_{p}(\boldsymbol{x})$, каждый из которых равен 1 в узле, соответствующем индексу $p$, равен 0 во всех остальных узлах сетки и в каждой ячейке сетки является билинейной (линейной по каждой переменой) функцией.

Решение в каждой подобласти будем аппроксимировать линейной комбинацией

$$
\boldsymbol{u}(t, \boldsymbol{x})=\sum_{p} H_{p}(\boldsymbol{x}) \boldsymbol{u}^{p}(t)
$$

Тогда задача построения приближенного решения начально-краевой задачи для системы уравнений (27) с условиями (28) на внутренних границах на каждом временном слое сводится к нахождению значений в узлах $\boldsymbol{u}^{p}\left(t_{m+1}\right)$ при известных значениях на предыдущем временном слое $\boldsymbol{u}^{p}\left(t_{m}\right)$.

Для внутренних узлов каждой из подобластей значения на следующем временном слое находим в соответствии с формулами

$$
\boldsymbol{u}\left(t_{m+1}, \boldsymbol{x}\right)=\sum_{\boldsymbol{k}} \boldsymbol{C}_{\boldsymbol{k}} \boldsymbol{u}\left(t_{m}, \boldsymbol{x}-\boldsymbol{\lambda}_{\boldsymbol{k}} \tau\right)
$$

В правой части формулы (31) отсутствуют слагаемые, связанные с условиями на внутренних и внешних границах. Эти слагамые равны 0 в соответствии с замечанием 2.2. Действительно, в силу условия (30) выбора шага по времени прямая линия $\frac{d \boldsymbol{x}}{d t}=\boldsymbol{\lambda}$, проведенная через точку $\left(t_{m+1}>0, \boldsymbol{x}\right)$, пересекает и внутренние и внешние границы в момент времени $t^{*}<t_{m}$.

Для узлов, лежащих на внешних границах, на которых поставлены «прозрачные» граничные условия, значения на следующем временном слое вычисляем как решение задачи Римана с «прозрачными» граничными условиями (26).

Для узлов на внешних границах, на которых поставлены «свободные» граничные условия, значения на следующем временном слое вычисляем как решение задачи Римана с граничными условиями (25). В качестве условий на границе следует брать равенства (29).

На основании изложенного выше был построен вычислительный алгоритм и реализован в системе MATLAB. Проведенные расчеты показали высокую эффективность этого алгоритма. Сравнительные тесты с другими вычислительными алгоритмами решения рассматриваемого класса задач, в частности, с вероятно наиболее эффективным и популярным из них - разрывным методом Галеркина - показали, что при заданной необходимой точности время расчета и необходимые требования по памяти существенно меньше. Поскольку решение на следующем временном слое в каждом узле сетки вычисляется независимо, построенный алгоритм обладает большим потенциалом к распараллеливанию.

Выполненные расчеты позволили подтвердить гипотезу, что наличие трещин в геологической породе, в которых соприкасающиеся части могут смещаться друг относительно друга, может приводить к тому, что упругая волна, сгенерированная виброисточником, не рассеивается по полусфере, а распространяется достаточно узким пучком так, что и на существенных глубинах ее плотность энергии остается значимой, чтобы вызвать те или иные физико-химические процессы. Внутренние границы (трещины) ведут себя как стенки волновода. Упругая волна практически не проходит сквозь них, и возмущение источника достигает значительной глубины без 
рассеяния. Эти и другие результаты вычислительных экспериментов будут подробно изложены в последующих публикациях.

7. Заключение. В работе для гиперболических систем линейных дифференциальных уравнений первого порядка с постоянными коэффициентами, с произвольным количеством пространственных переменных построено приближенное решение обобщенной задачи Римана с условиями сопряжения на разрыве. Также приведено приближенное решение обобщенной задачи Римана с граничными условиями. Для этого построено фундаментальное решения оператора задачи. Это, в свою очередь, позволило свести задачу Римана к решению системы алгебраических уравнений с правой частью, зависящей от значений переменных в начальный момент времени в конечном числе точек.

На основе этих решений задачи Римана построен и реализован вычислительный алгоритм нахождения решения начально-краевой задачи для гиперболических систем линейных дифференциальных уравнений первого порядка с постоянными коэффициентами. При этом постановка задачи допускает существование внутренних границ, на которых решение может иметь разрывы значений переменных модели и должны выполняться заданные уловия, связывающие значения переменных по обе стороны этих границ.

Построенный вычислительный алгоритм был применен для исследования характера распространения упругих волн, сгенерированных периодически действующим виброисточником, в блочно-трещиноватой геологической среде. Существование трещин отражено в модели наличием внутренних границ, на которых выполняются «условия проскальзывания без трения». Проведенные численные эксперименты показали высокую эффективность этого вычислительного алгоритма и его потенциал, как инструмента для исследования практических прикладных задач.

\section{СПИСОК ЛИТЕРАТУРЫ}

1. Владимиров В. С. Уравнения математической физики. - М.: Наука, 1981.

2. Гельфанд И. М., Шилов Г. Е. Некоторые вопросы теории дифференциальных уравнений. - М.: ГИФМЛ, 1958.

3. Гельфанд И. М., Шилов Г. Е. Обобщенные функции и действия над ними. - Мю: ГИФМЛ, 1959.

4. Гельфанд И. М., Шилов Г. Е. Пространства основных и обобщенных функций. - М.: ГИФМЛ, 1958.

5. Куликовский А. Г., Погорелов Н. В., Семенов А. Ю. Математические вопросы численного решения гиперболических систем уравнений. - М.: Физматлит, 2001.

6. Скалько Ю. И. Задача Римана о распаде разрыва в случае многих пространственных переменных// Тр. МФТИ. - 2016. - 8, № 4. - С. 169-182.

7. Скалько Ю. И. Корректные условия на границе, разделяющей подобласти// Комп. исслед. модел. 2014. - 6, № 3. - C. 347-356.

8. LeVeque R. L. Finite Volume Methods for Hyperbolic Problems. - Cambridge: Cambridge Univ. Press, 2002.

9. Kaser M., Dumbser M. An arbitrary high order discontinuous Galerkin method for elastic waves on unstructured meshes. I. The two-dimensional isotropic case with external source terms// Geophys. J. Int. 2006. - 166. - P. 855-877.

Скалько Юрий Иванович

Московский физико-технический институт

E-mail: skalko@mail.mipt.ru

Гриднев Сергей Юрьевич

Воронежский государственный технический университет

E-mail: gridnev_s_y@rambler.ru 